\title{
Electric Power Saving Awareness System at School Using ICT
}

\author{
Koichi Kuzume ${ }^{\mathrm{a}}$, Tomonori Tabusa and Haruka Sawa \\ Information Science and Technology Department, National Institute of Technology, Yuge College,1000 Kamishima- \\ cho, Ehime-ken, Japan
}

\begin{abstract}
Nowadays, education on energy saving at schools attracts attention to reduce energy consumption. In this paper we proposed a novel system to promote energy conservation activity at schools using a sensor network. The system consisted of a wall-type clock embedded with several kinds of sensors for temperature and light in the classroom, and wireless devices to connect to a cloud computer network. The system had the following superiorities: offered various services for energy conservation activity, needless of CT (Current Transformer) sensor, low price, ease of installation and extension, and visualization of power consumption in conjunction with school timetable in real time. We presented the system and a user study conducted to evaluate its usefulness.
\end{abstract}

\section{Introduction}

In order to reduce electric power consumption, it is necessary to examine our lifestyles and develop awareness of electric power conservation. It has been pointed out that activities to enlighten people on power saving or energy conservation are very important. Educational institutions, such as primary schools and junior and senior high schools, appeal as organizations particularly suited to teaching energy conservation. Many researchers have already reported on energy conservation at schools[1-2]. Some schools set up a smart meter, displaying electric power consumption, allowing school children to check power usage in real time. They could identify potential for saving power in their schools, by becoming aware of energy consumed through their actions. Communication between people plays an important role in deepening understanding of energy saving. Miura reported on the usefulness of the power meter for electric power saving activity [2]. In elementary schools, power saving activity by children mainly involved turning on or off lights and air-conditioners in classrooms and toilets. The amount of energy consumed in school was not so large. However, school children spoke at home of their experiences in school, including the energy conservation campaign. As a result of their action, the energy conservation campaign could be expected to have had a wider and wider impact in the school zone or community in which the children lived. If energy saving activity or education on global warming were more advanced in schools, we could realize huge savings in energy consumption beyond our forecasts.

Visualization technology for power consumption is very effective to promote energy conservation. There have been developed a lot of visualization systems. Florian and Kevin developed a system

\footnotetext{
${ }^{a}$ Corresponding author : kuzume@info.yuge.ac.jp
} 
called Ubiquitous Smart Energy Management (USEM), which combined home automation and energy usage monitoring technology [3]. The system made use of Current Transformer (CT) sensors to measure the current of electric appliances. It was necessary to attach it to all electric appliances intended to be monitored for power consumption and to connect to a sensor network to send the data. However, neither smart meter nor USEM are suitable for promoting power conservation activity in schools because USEM needs a lot of sensors to measure the electric current of each appliance and they are expensive in terms of construction work.

In a previous paper, we proposed a novel system for promoting energy saving activity in schools, without $\mathrm{CT}$ devices, with ease of construction and low price, and confirmed its basic operation [1].In this paper we review our modified system and present experimental results from a user study to confirm its usefulness.

This paper is organized as follows: In section 2, we present the concept of the system proposed and a novel visualization technology for electric power consumption using a sensor network called Eco Assist Clock (EAC). Then the architecture of the EAC and its networking are described. In section 3, we detail the support service for energy saving activity in conjunction with the school lecture timetable. Section 4 presents the results from a user study to evaluate the performance of the system. Finally, section 5 outlines our conclusions and future work.

\section{Electric Power Saving Awareness System}

\subsection{Architecture of Electric Power Saving Awareness System}

Figure1 shows the architecture of the electric power-saving awareness system we proposed. A clock was installed, containing several kinds of sensors, such as for temperature, light and wind, to monitor the amount of energy consumed in a classroom. The energy saving support system at school consisted of the sensor network constructed from several EACs, the database for storing data and the management system offering several services to promote energy saving activity. The EACs, which could sense the on/off switching state of the lights and air-conditioners, sent the data to the management PC via Zigbee wireless devices [4]. The data collected could be stored on the management PC and also immediately sent to the Cloud Web Server. The accumulated data could be visualized on the web site. School children and teachers could access the web and check the power usage situation from anywhere in real time. School children could brow seanytime and anywhere, including with their families, and communicate with their families or their communities about events at schools using the internet. This could help promote energy conservation activity. The software for developing our system was as follows: MySQL was used for the database system, Apache for constructing the web server, and PHP for web programming. Visualization of power consumption on the web was realized using the graphic library provided by PHP.

\subsection{Eco Assist Clock}

Smart meters have usually been utilized for visualization of power consumption. They have many functions, not only the display or reporting of power consumption, but also the control of electric appliances. Suchun necessary functions and high installation cost are not needed for promoting power conservation in schools. We proposed a novel device, ECA, for visualization of power consumption. Figure 2 and 3 show a circuit diagram for the EAC and its prototype model. The clock used in our system was a commercial clock of $26 \mathrm{~cm}$ in diameter, costing 5,500 yen. It was installed in the most conspicuous place in the classroom, on the wall above the blackboard. The EAC contained light, temperature and wind sensors, LEDs, a wireless module, and a microcomputer (Arduino) [5] embedded in the clock. The reason for adopting a wall-type clock was that school children looked at it frequently during lectures. The clock embedded in the EAC ran independently from that of the clock itself. Moreover, it warned students of energy waste by flashing the LED lights. 


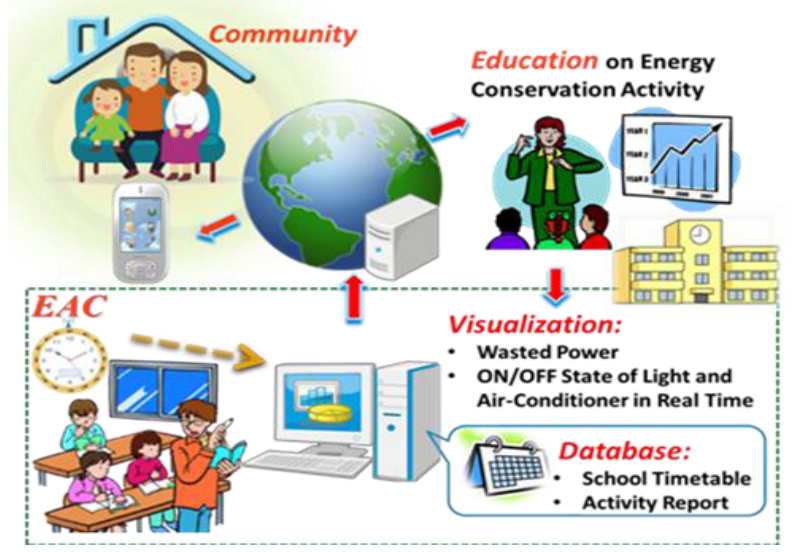

Figure 1. Architecture of Electric Power Saving Awareness System.
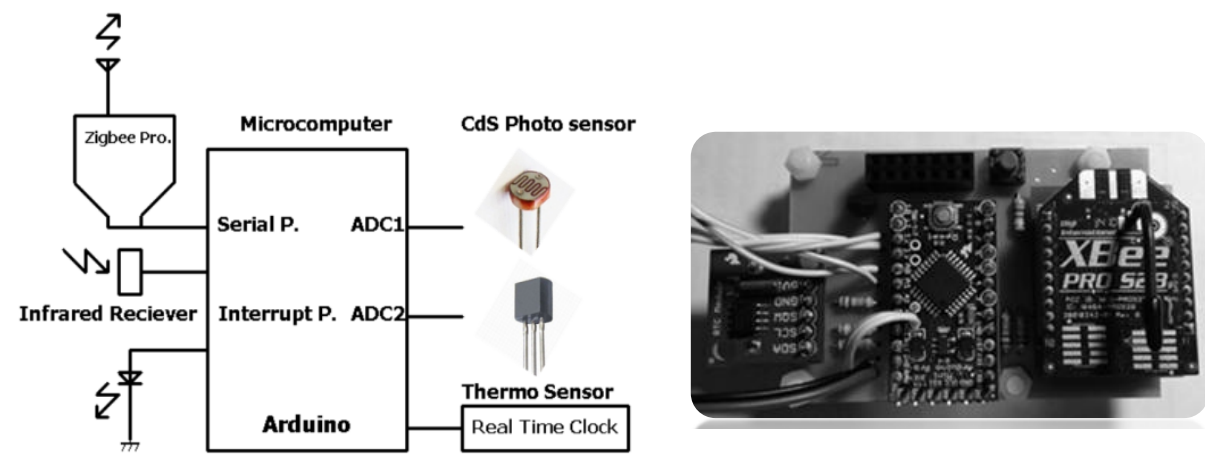

Figure 2. Architecture of EAC and Fabricated Print Circuit Board.

\subsubsection{Detection of ON/OFF Switching of Lights and Air-Conditioner in Classroom}

A CdS-photo sensor was used to detect the on/off switching state of the lights. It was fabricated at the top of the clock. It was not necessary to accurately measure power consumption, approximate power consumption being estimated from the on/off switching data. There are 24 fluorescent lights in the classroom and each light has $30 \mathrm{~W}$ power consumption. Therefore, the power consumption is about $0.72 \mathrm{~kW}(24 \times 30 \mathrm{~W})$. The wind sensor can detect the air movement generated by an air-conditioner. The on/off signal of the air-conditioner is sent to the EAC by an infrared transmitter. The wind sensor could detect air movement generated by an air-conditioner. The on/off signal of the air-conditioner was sent to the EAC via infrared.

\subsubsection{Data Transmission by Wireless Communication}

Useful information, such as the temperature, the on/off switching state of the lights and air-conditioner etc. were sent to the personal computer via a wireless communication device, Zigbee Pro., which could communicate with the base station $1.5 \mathrm{~km}$ distance from the EAC outside. The reason for using the wireless network was that it could extend the area of installation toother places in the campus area with lower construction cost. 


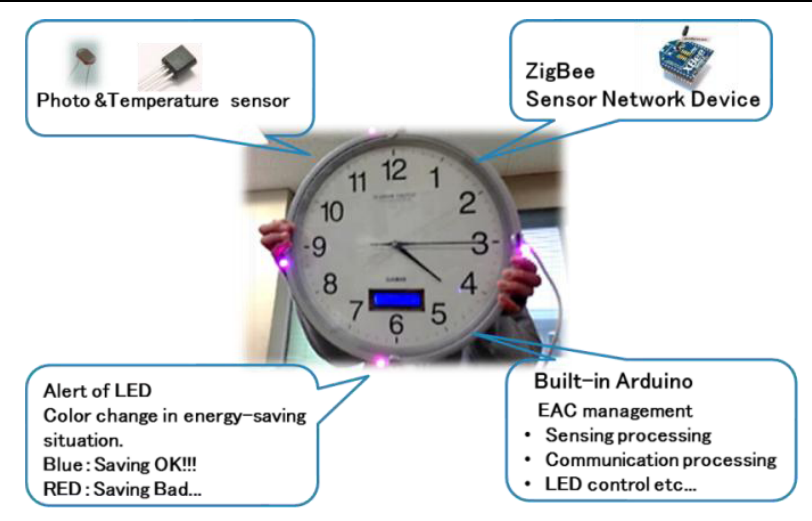

Figure 3. Prototype of Eco Assist Clock.

\section{Support Service for Energy Saving Activity}

\subsection{Evaluation of Power Saving Activity in Conjunction with School Timetable}

Figure 4 shows the top webpage. This page contained the following information. (i) Power consumption of both classrooms and whole school. (ii) On/off switching state of lights in classrooms installed with the EAC. If the lights were turned on, the picture of the light on the web changed to yellow. (iii) A report on power consumption and wasted power consumption over a day, a week or a month, could be displayed. Wasted power consumption could be calculated and the time and duration when the lights in the classroom were turned on applied in red to the school lecture timetable. See Figure 5 .

\subsection{Definition of Performance Factor of Power-saving Activity}

At primary schools and junior and senior high schools in Japan, school children are assigned to learn in a set classroom. They usually study in their classroom, except for special subjects, like experiments and gymnastics, etc. We introduced the performance factor for evaluation of power-saving activity below,

$$
P F_{\text {save }}=\frac{\sum T_{p}}{\sum T_{\text {out }}} \times 100[\%]
$$

where $T_{p}$ is energy saving interval time and $T_{\text {out }}$ is time period when the classroom was unoccupied. $T_{p}$ could be measured by our system and $T_{\text {out }}$ could be ascertained from the school lecture timetable. If all lights in the classroom were turned off while school children were outside the classroom, the powersaving factor, $P F_{\text {save }}$, was equal to $100[\%]$. This factor could be utilized for evaluating energy saving activity. The web page could be browsed by a mobile phone, as shown in Figure 6. The result of measured power consumption and $P F_{\text {save }}$ over 6 days are shown in Figure 7.

\section{User Study for Evaluation of System Performance}

\subsection{Methodology and Study Participants}

The $1^{\text {st }}$ grade, 46 students of our college's Information Science and Technology Department, participated in the study. First we set an EAC on the wall over the blackboard in the classroom without giving notice and monitored power consumption over a week. Next we explained about our study and showed our webpage. We asked them to check the web site using their mobile phones more 
than once a day during the $2^{\text {nd }}$ week. We then compared $P F_{\text {save }}$ for the two periods. Finally, after the completion of the test, the participants were asked to complete a questionnaire.

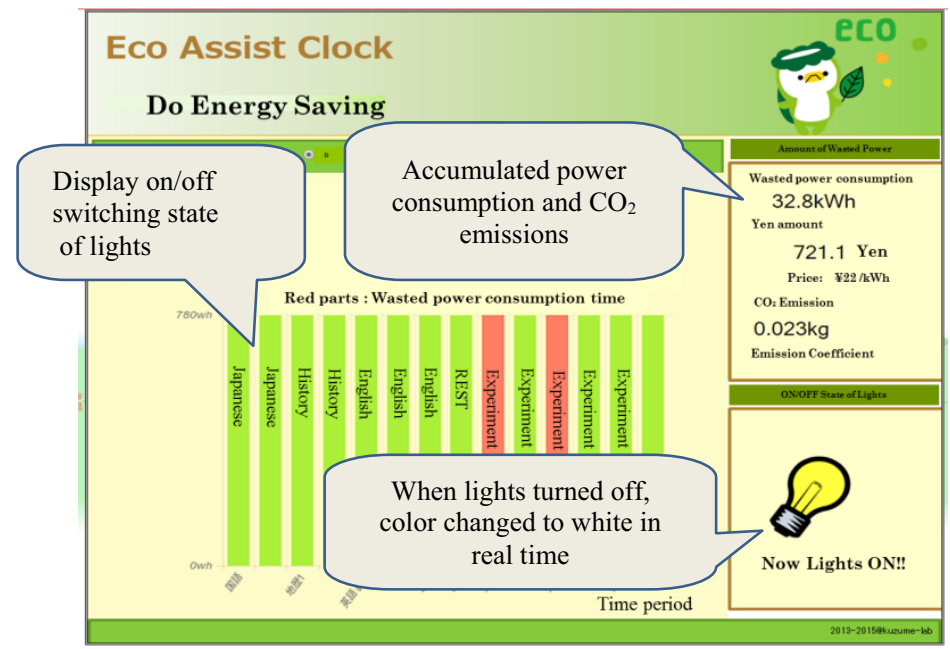

Figure 4 Top Page of the Power Saving Activity Support System

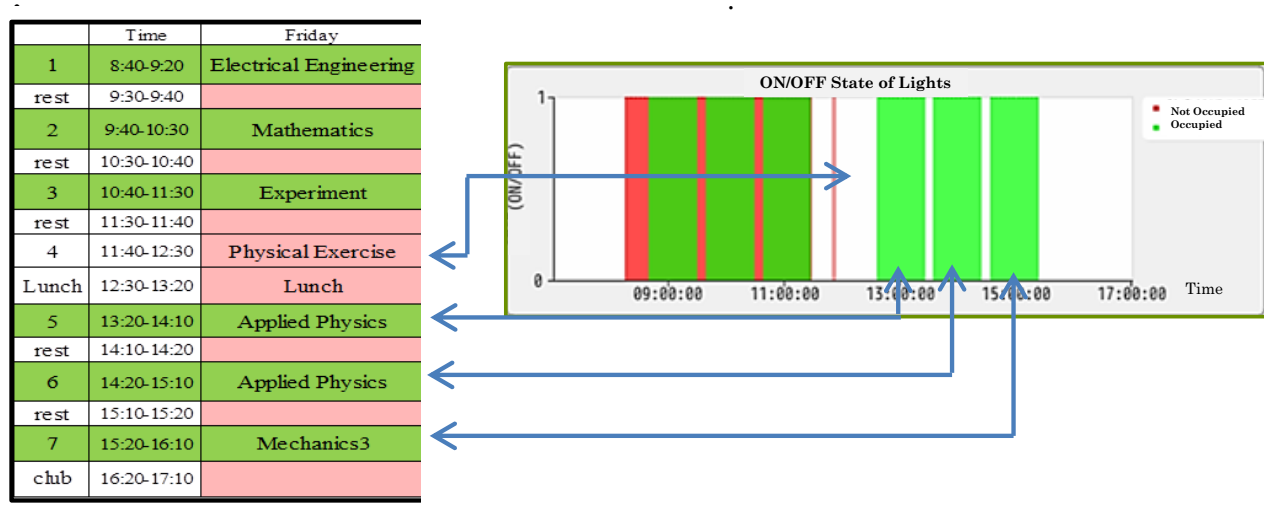

Figure 5. Wasted Power Consumption in Conjunction with School Timetable.

The principal questions of the questionnaire were as follows:

Q1.How many times did you browse the web site over the test period?

Q2.How many times did you turn off the lights in your classroom over the test period?

Q3.Have you ever studied how to conserve energy? If yes, when and where?

Q4.How likely do you think it would be that students would conduct energy conservation activity with the help of the visualization on the mobile phone?

Q5.What is the most useful content displayed on web site to encourage your energy-saving?

Q6.How useful do you find the overall system with regard to efficient energy usage?

Participants answered questions 5 and 6 using the Likert scale of 1-10, with 1 being the least positive and 10 the most positive.

\subsection{Result of User Study}

Figure 7 shows the performance factor, $P F_{\text {save }}$, over the week without notice. Average $P F_{\text {save }}$ was around $80 \%$. After notification of our test, average $P F_{\text {save }}$ increased slightly. Participants had already formed habits for energy conservation. According to answers in the questionnaire, since the occurrence of the Great East Japan Earth Quake, 70\% of participants had learned about power 
conservation as part of environmental education in junior high school (Q3). The number of students who voluntarily browsed the web site during the user test was less than $20 \%$ and only $10 \%$ of students turned off the light in the classroom (Q1,Q2). About 65\% of students answered that the flashing light of LEDs was the most useful method to let students know aboutenergy conservation.

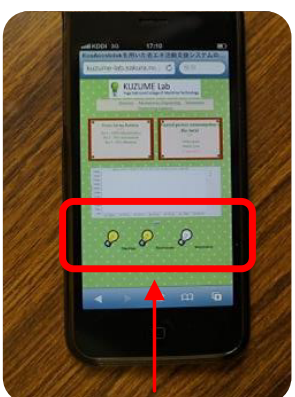

Display of state oflightsin real time Figure 6. Notification via Mobile Phone.

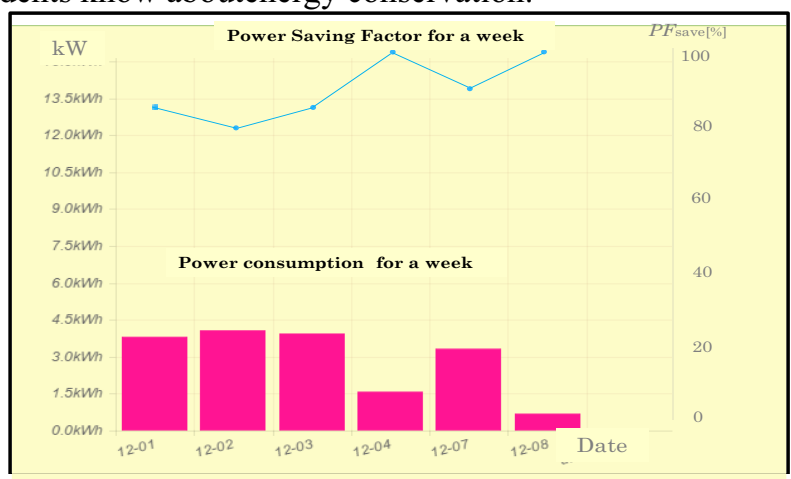

Figure 7. Report on Power Consumption over A Week.

(Likert value was over 7)(Q7).From content displayed on the web site, knowing the status of lights was utilized to prevent forgetting to turn off lights (over $80 \%$ of participant answered over 7 in Likert) $(\mathrm{Q} 5,6)$. The $90 \%$ of answers to question7 with over 7 in Likert, showed our system had potential use in energy conservation activity (Q4).

\section{Conclusions}

In this research we proposed an electric power saving awareness system at school using ICT and constructed a prototype EAC. To evaluate the system we conducted a user test and questionnaire. We measured the wasted power consumption over a week and compared the performance factor in two cases. One involved no user notification; while for the other users were notified about the test. According to the questionnaire, our system had potential benefit when conducting energy conservation activity. To improve the performance factor, the user needed more opportunities to recognize energy saving activity. In other words, it would be more effective to add functions, e.g. the interaction between man and the EAC. It is important to note which methods or systems could assist people in changing their behaviour.

\section{Acknowledgments}

This research was partly supported by Grant-in-Aid for Scientific Research No.25330221.

\section{References}

1. Koichi Kuzume and Masakazu Okada, "Sensor Network System to Promote Energy Conservation --Realization of Energy Smart School--”, The Proceedings of the IEEE International Conference on Pervasive Computer and Communications, Budapest (2013).

2. Shuichi Miura, "Practice and an effort of Environmental Education on Energy Conservation in an Elementary School", The Proceedings of the $22^{\text {nd }}$ Energy and Resource Research Meeting, pp275-278, June, (2003) (in Japanese).

3. Florian Reinhart et.al,"Fostering Energy Awareness in Residential Homes using Mobile Devices", The Proceedings of the Fourth International Conference on Smart Grids, Green Communications and IT Energy-aware Technologies, IARIA, pp.35-43 (2014).

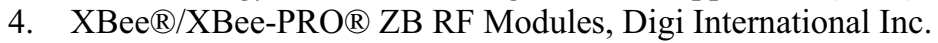

5. http://www.arduino.cc/. 